\title{
A survey on elliptic curve cryptography
}

\begin{abstract}
Cryptography is an evolving field that research into discreet mathematical equation that is representable by computer algorithm for providing message condentiality. The scheme has been widely used by nation-states, corporates and individual who seeks privacy for data in storage and during transmission. This paper provides a ground up survey on elliptic curve cryptography. It tailors the mathematics behind elliptic curve to the applicability within a cryptosystem. In brief, elliptic curve is a study of points on two-variable polynomials of degree 3. With curve dened over a finite field, this set of points acted by an addition operation forms a finite group structure. Also known as torsion points, they are used to represent the coded messages. Encryption and decryption transform a point into another point in the same set. Besides providing conceptual understanding, discussions are targeting the issues of security and efficiency of elliptic curve cryptosystem. This paper serve as a basis in guiding anyone interested to understand the fundamental concept behind this cryptosystem. Moreover, we also highlight subareas of research within the scope of elliptic curve cryptosystem.
\end{abstract}

Keyword: Elliptic curve; Endomorphism; Finite field; Group structure; Scalar multiplication 